\title{
En hipertensos tratados, el monitoreo ambulatorio fue un buen predictor de riesgo cardiovascular
}

Risk of Cardiovascular Disease in Relation to Acchieved Office and Ambulatory Blood Pressure Control in Treated Hypertensive Subjects. Verdecchia P, Reboldi G, Porcellati C et al. J Am Coll Cardiol 2002;39:878-85.

\section{Objetivo}

Evaluar el impacto pronóstico del monitoreo ambulatorio de la presión arterial de 24 hs (MAPA) en hipertensos bajo tratamiento.

\section{Diseño}

Estudio de cohorte prospectivo*

\section{Lugar}

Perugia, Italia

\section{Métodos}

Como parte del estudio PIUMA, se realizaron evaluaciones diagnósticas que incluían el MAPA, a pacientes hipertensos antes de iniciar tratamiento, y luego de un promedio de 3.7 años de seguimiento. Luego de esto se evalúo el desarrollo de eventos cardiovasculares en el seguimiento.

\section{Pacientes}

Se incluyeron 790 sujetos con hipertensión arterial esencial (HTA) con un promedio etario de 48 años.

\section{Medición de resultados}

Evaluación de la utilidad de distintas variables para predecir un primer evento vascular ( infarto de miocardio o angina inestable con alteraciones del ECG o revascularización coronaria o angioplastía o muerte súbita cardíaca o insuficiencia cardíaca que requiera internación o accidente cerebrovascular o ataque isquémico transitorio).

\section{Resultados}

Al final del seguimiento, $26 \%$ de los sujetos se hallaban adecuadamente controlados en el consultorio $(<140 / 90 \mathrm{mmHg})$; y estos fueron un $37.3 \%$ basándose en el MAPA (PAdiurna $<135 / 85 \mathrm{mmHg}$ ). De los 790 pacientes iniciales, sufrieron eventos cardiovasculares luego de la visita de seguimiento 58 pacientes. El control a través del MAPA predijo un menor riesgo cardiovascular subsecuente independientemente de otros factores de riesgo individuales. El control evaluado en el consultorio también se asoció a un menor riesgo cardiovascular, pero no fue estadísticamente significativo (ver tabla). Por ejemplo, la tasa de eventos cardiovasculares fue de 0.71 cada 100 pacientes/año en los controlados por MAPAvs. 1.87 en los que según el MAPAno lo estaban.

Tabla: Utilidad pronóstica del adecuado control medido en consultorio y a través del MAPA

\begin{tabular}{l|c|c}
\hline Variable & RR (IC 95\%) & $\mathbf{p}$ \\
\hline Control adecuado en el consultorio & $0.64(0.31$ a 1.61) & NS \\
\hline Control adecuado en el MAPA & $0.36(0.18$ a 0.70) & 0.003 \\
\hline
\end{tabular}

Las variables consideradas en el análisis multivariable* incluyeron edad, diabetes, hipertrofia de $\mathrm{VI}$, y presiones pretratamiento e intratratamiento.

\section{Conclusiones}

El control evaluado a través del MAPA fue superior al realizado en el consultorio para predecir el riesgo cardiovascular en pacientes tratados con HTA.

Fuente de Financiamiento: no referida

\section{Comentario}

Siendo la hipertensión arterial uno de los problemas de salud acerca de los que más se investiga y se trata a nivel mundial, llama la atención el pobre desempeño de casi todos los sistemas evaluados: solo una minoría de los hipertensos se diagnostican, y de los diagnosticados solo unos pocos se encuentran adecuadamente controlados ${ }^{1}$. No es cuestión de buscar un culpable (si el médico poco agresivo, el paciente poco adherente, etc.). El tema es que el "sistema" actual funciona pobremente. La falta de control adecuada de la HTA es una de las piezas importantes a evaluar y mejorar en este proceso: se sabe que el mal control es un importante predictor de morbimortalidad cardiovascular en hipertensos ${ }^{2}$.

Hasta la aparición del monitoreo ambulatorio de presión arterial (MAPA), los criterios de control adecuado se basaron principalmente en la TAde consultorio. Algunos estudios indican que las mediciones del MAPA son algo mejores que las del consultorio para la estratificación del riesgo cardiovascular en $\mathrm{HTA}^{3,4}$. La particularidad del presente estudio de Verdecchia y cols. es que agrega información acerca de la utilidad del MAPA para evaluar el "buen control" en pacientes de la comunidad con HTAbajo tratamiento.

Esta claro que la TA lograda con el tratamiento es un predictor muy importante de riesgo cardiovascular en hipertensos tratados. En este estudio los autores concluyen que el control de la TAmedido a través del MAPA fue mejor predictor que el medido en el consultorio. No obstante, no se pude concluir que el MAPA sea mejor que el con- sultorio dado que sus intervalos de confianza se superponen y no hay suficiente poder* para evaluar el control por consultorio.

Otra limitación del estudio es su amplia definición de "evento" (desde muerte súbita cardíaca hasta ataque isquémico transitorio).

Como concluyen los autores, sus resultados no implican la utilización del MAPA de rutina en todo hipertenso. Su rol se centra en algunas situaciones particulares como la HTA de guardapolvo blanco, y tal vez en sujetos con excesiva variabilidad de la PA, con síntomas sugestivos de hipotensión, y en la aparente resistencia a múltiples drogas $^{5,6}$. Un estudio que comparó estrategias de seguimiento de la HTA basadas en la TA de consultorio vs. en el MAPA no mostró distintos resultados clínicos. La única diferencia fue el menor uso de drogas en el grupo MAPA ${ }^{7}$. Es preciso ahora que ensayos clínicos que comparen la morbimortalidad en HTAcomparen tres estrategias de control: la clásica (basada en el consultorio), aquella basada en el MAPA, y también la basada en el automonitoreo domiciliario de la TA.

Conclusiones del comentador: El control de la presión es un predictor de eventos cardiovasculares, tanto medido a través del MAPAcomo en el consultorio. Dado que no hubo suficiente poder ${ }^{*}$ para evaluar el control de consultorio, no se puede afirmar que uno sea superior a otro.

Dr. Federico Augustovski [ Unidad de Medicina Familiar y Preventiva. Hospital Italiano de Buenos Aires ]

\section{Referencias}

1. Rubinstein A. Hipertensión Arterial. En: PROFAM: Programa de Educación a Distancia de Medicina Familiar y Ambulatoria / Esteban Rubinstein, dir. Segunda edición. Buenos Aires: Hospital Italiano de Buenos Aires. Departamento de Docencia e Investigación, c2001. v.3, p.53-117.

2. The IPPPSH Collaborative Group. Cardiovascular risk and risk factors in a randomized trial of treatment based on the beta-blocker oxprenolol: the International Prospective Primary Pre2. The IPPPSH Collaborative Group. Cardiovascular risk and risk factors in a

3. Verdecchia P. Prognostic value of ambulatory blood pressure : current evidence and clinical implications. Hypertension. 2000 Mar;35(3):844-51

4. Mancia G, Parati G. Ambulatory blood pressure monitoring and organ damage. Hypertension. 2000 Nov;36(5):894-900

5. The sixth report of the Joint National Committee on prevention, detection, evaluation, and treatment of high blood pressure. Arch Intern Med. 1997 Nov 24;157(21):2413-46.

6. 1999 World Health Organization-International Society of Hypertension Guidelines for the Management of Hypertension. Guidelines Subcommittee. J Hypertens. 1999 Feb;17(2):151-83. 7. Staessen JA, Byttebier G, Buntinx F, Celis H, O'Brien ET, Fagard R. Antihypertensive treatment based on conventional or ambulatory blood pressure measurement. A randomized controlled trial. Ambulatory Blood Pressure Monitoring and Treatment of Hypertension Investigators. JAMA. 1997 Oct 1;278(13):1065-72. 